\title{
Fungal infections of the central nervous system and paranasal sinuses in onco-haematologic patients. Epidemiological study reporting the diagnostic-therapeutic approach and outcome in 89 cases
}
A. Candoni ${ }^{1}$
N. Klimko ${ }^{2}$
A. Busca ${ }^{3}$
R. Di Blasi ${ }^{4}$
O. Shadrivova ${ }^{2}$
S. Cesaro ${ }^{5}$
M. E. Zannier ${ }^{1}$
L. Verga6
F. Forghieri $^{7}$ ।
E. Calore ${ }^{8}$
G. Nadali ${ }^{9}$
E. Simonetti ${ }^{10}$
P. Muggeo ${ }^{11}$
A.M. Quinto ${ }^{12}$
C. Castagnola ${ }^{13}$
M. Cellini ${ }^{14}$
M.I. Del Principe ${ }^{15}$
N. Fracchiolla ${ }^{16}$
L. Melillo ${ }^{17}$
M. Piedimonte ${ }^{18}$
D. Zama ${ }^{19}$
F. Farina ${ }^{6}$
D. Giusti ${ }^{7}$
F. Mosna ${ }^{20}$ |
D. Capelli ${ }^{21}$
M. Delia ${ }^{22}$
M. Picardi ${ }^{23}$
N. Decembrino ${ }^{24}$
K. Perruccio ${ }^{25}$ ।
S. Vallero ${ }^{26} \mid$ F. Aversa ${ }^{27} \mid$ R. Fanin ${ }^{1}$ | L. Pagano ${ }^{4}$ on behalf of SEIFEM Group
(Epidemiological Surveillance of Infections in Haematological Diseases)

\footnotetext{
${ }^{1}$ Clinica Ematologica, ASUI, Udine, Italy

${ }^{2}$ Metchnikov North-Western State Medical University, St. Petersburg, Russia

${ }^{3}$ S.C. Ematologia, AO Città della Salute e della Scienza, Torino, Italy

${ }^{4}$ Istituto di Ematologia, Polo Onco-Ematologico Fondazione Policlinico A. Gemelli-IRCCS, Università Cattolica del Sacro Cuore, Roma, Italy

${ }^{5}$ Oncoematologia Pediatrica, AOUI, Verona, Italy

${ }^{6}$ Clinica Ematologica, Ospedale S Geraldo, Monza, Italy

${ }^{7}$ Clinica Ematologica, Dipartimento di Scienze Mediche-Chirurgiche, Università di Modena, Modena, Italy

${ }^{8}$ Clinica di Oncoematologia pediatrica, Dipartimento di Salute della Donna e del Bambino, Azienda Ospedaliero-Universitaria, Padova, Italy

${ }^{9}$ U.O.C. Ematologia, AOUI, Policlinico GB Rossi, Verona, Italy

${ }^{10}$ Ematologia, Ospedale SM Misericordia, Perugia, Italy

${ }^{11}$ Oncoematologia Pediatrica, AOUC Policlinico, Bari, Italy

${ }^{12}$ UO di Ematologia, Dipartimento di Medicina, AO di Padova, Padova, Italy

${ }^{13}$ Dipartimento Oncoematologico Fondazione, ICRRS Policlinico San Matteo, Pavia, Italy

${ }^{14}$ UO Pediatria, Dipartimento Materno-Infantile, AOU Policlinico, Modena, Italy

${ }^{15}$ Ematologia, Dipartimento di Biomedicina e Prevenzione, Università Tor Vergata, Roma, Italy

${ }^{16}$ UO Oncoematologia, Fondazione IRCCS Ca' Granda Ospedale Maggiore Policlinico, Milano, Italy

${ }^{17}$ Divisione di Ematologia, IRCCS Casa Sollievo della Sofferenza, San Giovanni Rotondo, Italy

${ }^{18}$ Dipartimento di Clinica e di Medicina Molecolare, AOU Sant'Andrea, Università Sapienza, Roma, Italy

${ }^{19}$ Oncoematologia Pediatrica, Ospedale Sant Orsola Malpighi, Bologna, Italy

${ }^{20}$ Struttura Complessa Ematologia, Ospedale Ca' Foncello, Treviso, Italy

${ }^{21}$ Clinica Ematologica, Ospedali Riuniti di Ancona, Ancona, Italy

${ }^{22}$ Sezione di Ematologia, Università degli studi di Bari, Bari, Italy

${ }^{23}$ Dipartimento di Scienze Biomediche Avanzate, Università Federico II, Napoli, Italy

${ }^{24}$ Oncoematologia Pediatrica, Fondazione IRCCS, Policlinico San Matteo, Pavia, Italy

${ }^{25}$ Oncoematologia Pediatrica, Ospedale SM Misericordia, Perugia, Italy

${ }^{26}$ Ematologia Pediatrica, Ospedale Infantile Regina Margherita S. Anna, Torino, Italy

${ }^{27}$ Ematologia e Centro Trapianti Midollo Osseo, Università di Parma, Parma, Italy
} 
Correspondence

Anna Candoni, MD, Clinica Ematologica, Centro Trapianti e Terapie Cellulari, Azienda Sanitaria Universitaria Integrata di Udine, Udine, Italy.

Email: anna.candoni@asuiud.sanita.fvg.it

\section{Summary}

Invasive fungal infections (IFI) of the Central Nervous System (IFI-CNS) and Paranasal Sinuses (IFI-PS) are rare, life-threatening infections in haematologic patients, and their management remains a challenge despite the availability of new diagnostic techniques and novel antifungal agents. In addition, analyses of large cohorts of patients focusing on these rare IFI are still lacking. Between January 2010 and December 2016, 89 consecutive cases of Proven (53) or Probable (36) IFI-CNS (71/89) and IFI-PS $(18 / 89)$ were collected in 34 haematological centres. The median age was 40 years (range 5-79); acute leukaemia was the most common underlying disease (69\%) and $29 \%$ of cases received a previous allogeneic stem cell transplant. Aspergillus spp. were the most common pathogens (69\%), followed by mucormycetes (22\%), Cryptococcus spp. (4\%) and Fusarium spp. (2\%). The lung was the primary focus of fungal infection (48\% of cases). The nervous system biopsy was performed in $10 \%$ of IFI-CNS, and a sinus biopsy was performed in $56 \%$ of IFI-PS $(P=0.03)$. The Galactomannan test on cerebrospinal fluid has been performed in $42 \%$ of IFI-CNS (30/71), and it was positive in 67\%. Eighty-four pts received a first-line antifungal therapy with Amphotericine $B$ in $58 \%$ of cases, Voriconazole in $31 \%$ and both in $11 \%$. Moreover, $58 \%$ of patients received 2 or more lines of therapy and $38 \%$ were treated with a combination of 2 or more antifungal drugs. The median duration of antifungal therapy was 60 days (range 5 -835). A surgical intervention was performed in $26 \%$ of cases but only $10 \%$ of IFICNS underwent neurosurgical intervention. The overall response rate to antifungal therapy (complete or partial response) was 57\%, and 1-year overall survival was 32\% without significant differences between IFI-CNS and IFI-PS. The overall mortality was $69 \%$ but the IFI attributable mortality was 33\%. Mortality of IFI-CNS/PS remains high but, compared to previous historical data, it seems to be reduced probably due to the availability of newer antifungal drugs. The results arising from this large contemporary cohort of cases may allow a more effective diagnostic and therapeutic management of these very rare IFI complications in haematologic patients.

\section{KEYWORDS}

Aspergillosis, fungal CNS infectious, haematological diseases, leukaemia, neurologic complications, Zygomicosis

\section{1 | INTRODUCTION}

Invasive fungal infections (IFI) of the Central Nervous System (IFI-CNS) and those of the Paranasal Sinuses (IFI-PS) are rare, lifethreatening infections in haematologic patients, and their management remains a challenge despite the availability of new diagnostic techniques and novel antifungal agents. ${ }^{1-8}$ There is a lack of recent epidemiologic studies with a proper number of cases reporting the outcome of haematologic patients with IFI involving CNS and PS, and therefore, the most appropriate diagnostic and therapeutic approach remain to be clearly defined. In fact, in the literature, we found only case reports or small retrospective studies, more often not recent. ${ }^{1,9-13}$ The most frequently reported pathogens were moulds and especially Aspergillus and Mucormycetes. ${ }^{1,2,6,14,15}$ The therapeutic management of these infectious complications is still very complex and heterogeneous, and recent studies on the efficacy of new antimycotic drugs are limited so that there is no clear standard of care currently available. For these reasons, we decided to carry out a study providing an update on current epidemiology and outcome of IFI-CNS and IFI-PS occurring in haematological patients in recent years aiming to improve the knowledge and management of these serious and rare infectious complications. 


\section{2 | PATIENTS AND METHODS}

Between January 2010 and December 2016, we collected all cases of IFI-CNS and IFI-PS consecutively observed in 26 Italian haematology centres belonging to the SEIFEM group and in 8 Haematology centres of the St. Petersburg, Russia. The study was carried out after approval by the local ethics committees and the local health authorities.

The patients' privacy was preserved and each haematological centre reported an identifying code for each case. All the centres involved performed the similar diagnostic workup including microbiological [i.e, research of indirect biomarkers: serum $\beta$-D-Glucan, Galactomannan (GM) on blood, cerebrospinal fluid or other sites] and radiological investigations (i.e, computerised tomography [CT] and/ or magnetic resonance imaging [MRI]). For each case observed, a standardised Case Report Form (CRF) was filled out containing information regarding the haematological disease and its phase and status (induction, consolidation or salvage phase, remission, relapse or resistant disease status); risk factors for IFI (neutropenia and its severity, previous stem cell transplantation, Graft Vs Host Disease [GVHD], steroid therapy, diabetes, previous history of IFI, chemotherapy in the previous 30 days, type and length of antifungal prophylaxis); the characteristics of IFI (clinical symptoms, site of infection, positivity of direct and indirect tests, histological and cultural data, autopsy report); the therapy performed (including pharmacological and surgical treatment), the outcome of the infection and cause of death.

\section{1 | Definitions}

Severe neutropenia was defined as a number of granulocytes $(P M N) \leq 500 / \mathrm{mmc}$. Mild/moderate neutropenia was defined as PMN counts between 500 and 1500/mmc. Steroid therapy has been defined as the use of prednisone or prednisolone for at least 7 days at a dose of $25 \mathrm{mg} /$ day or $1 \mathrm{mg} / \mathrm{kg} /$ day, respectively. Fever has been defined according to the IDSA guidelines as an increase in body temperature $\geq 38.3^{\circ} \mathrm{C}$ once or $\geq 38^{\circ} \mathrm{C}$ for more than 1 hour.

Only the forms of Proven and Probable IFI were considered for this study. Each IFI case was defined as proven or probable according to the 2008 criteria established by the European Organization for Research and Treatment of Cancer/Mycoses Study Group (EORTC/ MSG). The IFI was declared disseminated when it involved 2 or more non-contiguous sites. ${ }^{16}$

Complete or partial response (CR, PR) to therapy was defined based on clinical, radiologic and/or mycological data as by the EORTC/MSG consensus group. Overall Response rate (ORR) was defined as CR+PR. ${ }^{16}$

In the case of a patient's death, the cause of death had to be specified as one of the following: (a) mycosis as primary cause, (b) haematological disease as primary cause with concomitant mycosis, (c) haematological disease in the absence of mycosis, (d) other causes.

We considered IFI attributable mortality every death occurring without evidence of active haematological disease and attributable only to infection as a primary cause of death.

\subsection{Statistical analysis}

Descriptive statistics (including mean, standard deviation, median, range, frequency, percentages) were calculated to analyse and compare the study cohorts. Overall survival (OS) (defined as the interval between the date of IFI diagnosis and the date of last follow-up or death) was estimated by the Kaplan-Meier survival analysis. OS curves were compared using the log-rank test. The independent effect of demographic/clinical predictors on OS was assessed by multivariate Cox proportional hazards regression analysis. Adjusted hazard ratios were computed, and 95\% confidence intervals $(\mathrm{Cl})$ for the hazard ratios and median OS time estimates are presented to assess the precision of the estimates obtained. All $P$-values are two-sided, with statistical significance evaluated at the 0.05 alpha level. Fisher's exact test was used to compare group differences in categorical variables. Data were analysed with MedCalc software (version 12.5.0.0; MedCalc Software bvba, Ostend, Belgium).

\section{3 | RESULTS}

\subsection{Characteristics of patients}

During the study period, 89 cases of Proven (53 cases) or Probable (36 cases) IFI with localisation to the CNS and PS were recorded in 34 Haematological centres. The median age of patients was 40 years (range 5-79). The study population included 23 (26\%) paediatric patients (age $\leq 18$ years). The demographic characteristics of patients and of the underlying haematological diseases at diagnosis are shown in Table 1.

The majority of patients had an acute myeloid (34\%) or lymphoid (35\%) leukaemia. Regarding the status of the haematological disease, most of the CNS/PS IFI has been documented in patients with an active haematological disease (81\%) and specifically at the onset of disease $(31 / 89,35 \%)$ or during its relapse $(41 / 89,46 \%)$. Twenty-nine per cent of patients (26/89) had previously received an allogeneic stem cell transplantation (Allo-SCT), in 16/26 cases from unrelated donors, in 5/26 from an HLA-identical family donor and in 5/26 from a haploidentical donor.

Most patients had neutropenia at the onset of IFI (74\%, 66/89) of severe grade in $49 \%$ of cases (44/89). Severe neutropenia has been reported more frequently in patients with IFI-PS $(P=0.0037)$. Patients received steroid therapy in $61 / 89$ cases $(69 \%)$, and 13 patients $(15 \%)$ had a history of diabetes.

\subsection{Clinical manifestations and aetiology of IFI}

The most common presenting symptoms were fever in 65\% (58/89) of cases and headache in $26 \%$ (23/89) of cases. Regarding neurological symptoms, seizures occurred in 16 cases (18\%) while in 43 cases (48\%) focal neurological symptoms (i.e, aphasia, dysarthria, diplopia) have been reported, with or without a concomitant alteration of consciousness. Neurological disorders were significantly more 
TABLE 1 Patients and haematologic disease characteristics. IFI characteristics

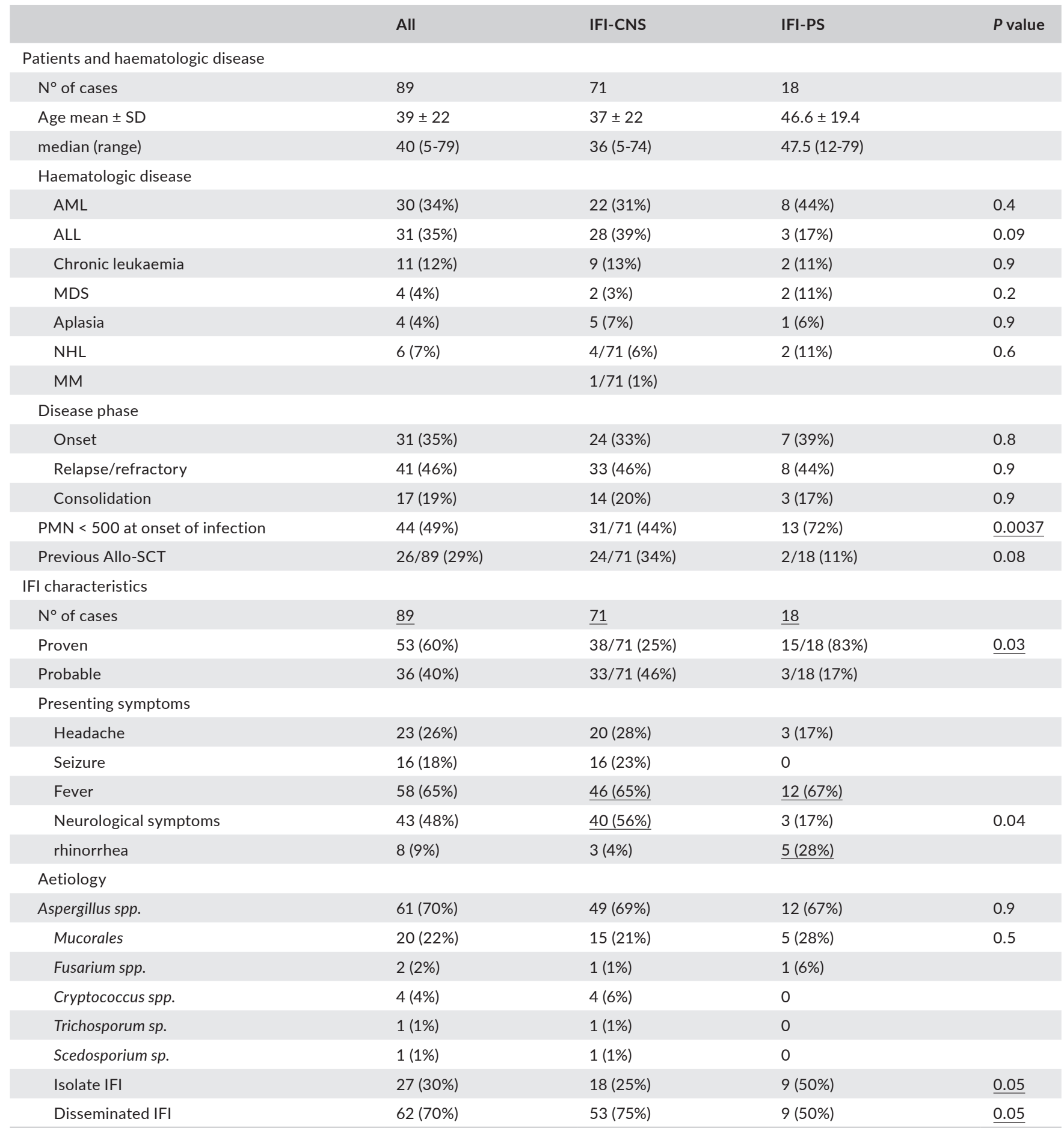

AML, acute myeloid leukaemia; ALL, acute lymphoid leukaemia; MDS, myelodysplastic syndrome, MM, multiple myeloma.

frequent in the IFI-CNS (40/71 vs 3/18). On the contrary, rhinorrhea was significantly more present in the IFI-PS (5/18 vs $3 / 71)$ (Table 1$)$.

\subsection{1 | Aetiological agents}

Proven infections were 53/89 (60\%), 48 caused by moulds and 5 by yeasts. Aspergillus spp. has been documented in 25 of proven cases, while the Mucorales in 20 cases. In 2 cases, the aetiologic agent was
Fusarium spp. and in 1 case Scedosporium apiospermum (Table 1). Proven IFIs caused by yeasts were 5: Cryptococcus spp. was isolated in most cases (4/5) while in one case the aetiologic agent was Trichosporon asahii. The probable infections were 36 (36/89, 40\%), all with Aspergillus aetiology. Overall, the majority of IFI were caused by Aspergillus spp. (proven + probable 61/89, 70\%). The specie was available in 19 cases: A. flavus was detected in 10 cases and A. fumigatus in 9 cases. 


\subsection{2 | Localisation of IFI}

There were $71 / 89$ cases (80\%) of CNS involvement (18/71-25\% with isolated localisation in the CNS) and $18 / 89$ cases (20\%) of PS involvement without concomitant CNS involvement (9/18 with isolated localisation of the paranasal sinuses). The PS and the CNS were both involved by contiguity in $15 / 89$ cases (17\%). The IFI was disseminated (involvement of 2 or more non-contiguous sites) in 62/89 cases (70\%) with significant prevalence in the cohort of IFI-CNS (53/71 cases$75 \%$, vs $9 / 18$ cases $-50 \% ; P=0.05$ ) (Table 1 ). The lungs have been identified as the primary focus of IFI in $48 \%$ of cases. Ten of 20 (50\%) cases of mucormycosis had only CNS and/or PS localisation.

\section{3 | Diagnosis of IFI}

The diagnosis was made by biopsy plus a histological examination in $26 / 89$ cases (29\%), with a significant higher rate of PS biopsies compared to CNS biopsies (18/26-69\% vs $7 / 26-26 \% ; P=0.005)$. In detail, in the IFI-CNS a biopsy of brain tissue was performed only in $10 \%$ of cases (7/71). The diagnosis of IFI was made with post-mortem examination in $6 \%$ of cases $(5 / 89)$.

Serum biomarkers coupled with radiological examinations (CT \pm MRI) have been used for diagnosis in $100 \%$ of cases. Coltures from infectious sites, blood or CSF, were performed in $44 / 89$ cases (49\%).
TABLE 2 Diagnostic procedures

\begin{tabular}{|c|c|}
\hline Histopathological examination & $26 / 89(29 \%)$ \\
\hline PS biopsy & $18 / 26(69 \%)$ \\
\hline CNS biopsy & $7 / 26(26 \%)$ \\
\hline Other site biopsy & $1 / 26(5 \%)$ \\
\hline \multicolumn{2}{|l|}{ Indirect biomarkers } \\
\hline GM test (Blood $\pm \mathrm{BAL})$ & $89 / 89(100 \%)$ \\
\hline Indirect biomarkers on CSF (IFI-CNS) & $34 / 71(48 \%)$ \\
\hline GM test on CSF & $30 / 71(42 \%)$ \\
\hline Positive GM test on CSF & $20 / 30(67 \%)$ \\
\hline Cryptococcus Capsular Antigen Test on CSF & $4 / 71(6 \%)$ \\
\hline Positive capsular antigen test on CSF & $4 / 4(100 \%)$ \\
\hline $\begin{array}{l}\text { Fungal coltures (from infectious sites, blood or } \\
\text { CSF) }\end{array}$ & $44 / 89(49 \%)$ \\
\hline Radiological examination (CT \pm MRI) & $88 / 89$ (99\%) \\
\hline Post-mortem diagnosis & $5 / 89(6 \%)$ \\
\hline
\end{tabular}

CSF, cerebrospinal fluid; BAL, bronchoalveolar lavage; CT, computerised tomography; MRI, magnetic resonance imaging.

The fungal biomarkers (GM, (1-3)- $\beta$-D-Glucan, cryptococcal capsular antigen) on cerebrospinal fluid (CSF) were performed in 34/71 cases of IFI-CNS (48\%). In all 4 cases of cerebral cryptococcosis, a
TABLE 3 IFI-therapy, response and outcome

\begin{tabular}{|c|c|c|c|c|}
\hline & ALL & IFI-CNS & IFI-PS & $P$ value \\
\hline $\mathrm{N}^{\circ}$ of cases & 89 & 71 & 18 & \\
\hline Drug therapy & $84(94 \%)$ & $66(93 \%)$ & $18(100 \%)$ & 0.57 \\
\hline \multicolumn{5}{|l|}{ Line of therapy } \\
\hline $1 \mathrm{td}$ & $35(42 \%)$ & $30(45 \%)$ & $5(28 \%)$ & \\
\hline$\geq 2 \mathrm{td}$ & 49 (58\%) & $36(55 \%)$ & $13(72 \%)$ & 0.28 \\
\hline Combination therapy & 32 (38\%) & $21(32 \%)$ & $11(61 \%)$ & 0.03 \\
\hline \multicolumn{5}{|l|}{ Length of therapy } \\
\hline mean \pm SD & $122 \pm 168$ & $131,8 \pm 185$ & $85,9 \pm 70$ & \\
\hline median (range) & $60(5-835)$ & $60(5-835)$ & $59(6-220)$ & \\
\hline Surgical resection & $22 / 84(26 \%)$ & $\begin{array}{l}\text { CNS surgery: } 7 / 66 \\
\text { (10\%) } \\
\text { Other sites surgery: } \\
9 / 66(14 \%)\end{array}$ & $\begin{array}{l}\text { PS surgery 6/18 } \\
(33 \%)\end{array}$ & 0.028 \\
\hline \multicolumn{5}{|l|}{ Response to treatment } \\
\hline ORR & $48(57 \%)$ & $36(54 \%)$ & $12(67 \%)$ & 0.57 \\
\hline $\mathrm{CR}$ & $29(34 \%)$ & $22(33 \%)$ & $7(39 \%)$ & 0.78 \\
\hline PR & $19(23 \%)$ & $14(21 \%)$ & $5(28 \%)$ & 0.54 \\
\hline NR & $36(43 \%)$ & $30(46 \%)$ & $6(33 \%)$ & 0.4 \\
\hline \multicolumn{5}{|l|}{ Outcome } \\
\hline Alive & $28(31 \%)$ & $23(32 \%)$ & $5(28 \%)$ & 0.77 \\
\hline Deaths & $61(69 \%)$ & $48(68 \%)$ & $13(72 \%)$ & \\
\hline Attributable mortality & $20 / 61(33 \%)$ & $14 / 48(29 \%)$ & $6 / 13(46 \%)$ & 0.32 \\
\hline Mortality for HD + IFI & $27 / 61$ (44\%) & $25 / 48(52 \%)$ & $2 / 13(15 \%)$ & 0.026 \\
\hline
\end{tabular}

$\mathrm{HD}$, haematologic disease; $\mathrm{ORR}$, overall response rate; $\mathrm{CR}$, complete response; $\mathrm{PR}$, partial response; NR, not response. 
CSF positivity of the cryptococcal capsular antigen was reported (4/4, 100\%). Galactomannan (GM) on CSF was tested in 30/71 (42\%) of IFI-CNS, and it was positive in $20 / 30$ (67\%) (Table 2).

\subsection{Antifungal treatment}

\subsection{1 | Antifungal prophylaxis}

Overall, 55 patients (62\%) received antifungal prophylaxis before the onset of IFI. In detail, 28/55 received Fluconazole (FLUCO), 14/55 Posaconazole (POSA), 5/55 Itraconazole (ITRA), 3/55 liposomal Amphotericin B (AMB), 3/55 Voriconazole (VORI), 1/55 Caspofungin (CASPO) and 1/55 Micafungin. The mean duration of antifungal prophylaxis was 18.5 days. None of the patients with a diagnosis of mucormycosis were receiving a polyene prophylaxis, and 12/61 (20\%) of cases with Aspergillosis (proven and probable) were receiving an Aspergillus active antifungal prophylaxis.

\subsection{2 | Antifungal therapy}

Table 3 shows the antifungal therapy administered to 84/89 patients for at least of 5 days. Forty-two per cent of the patients
(35/84) received only one line of therapy while $58 \%$ (49/84) 2 or more lines, without significant differences between IFI-CNS and IFI-PS. Combination therapy, with 2 or more antifungals, was administered in $38 \%$ of cases and was employed in a significant higher number of cases with PS involvement (32\% vs $61 \%, P=0.03$ ). The median duration of antifungal therapy was 60 days (range 5-835).

In detail, in the 49 cases of Aspergillosis of CNS, the antifungal agents most frequently used as first-line treatment were AMB (in 28 cases) and VORI (in 24 cases), alone or in combination. The 12 cases of Aspergillosis of PS were treated in the first line mainly with AMB ( 9 cases) and with VORI ( 3 cases); a combination therapy was administered only in 1 case. Of the 20 cases of Mucormycosis (15 CNS and 5 PS), 19/20 received a first-line antifungal therapy for at least 5 days: $A M B$ was administered to 9 patients, $A M B+P O S A$ to 8 patients, $A M B+C A S P O$ to 1 patient and POSA to 1 patient. All 4 cases of Cryptococcosis received FLUCO and AMB (3/4 cases), and the 2 cases of Fusariosis received AMB plus VORI.

Excisional surgery was performed in $26 \%$ of the cases (22/84), more often in patients with IFI-PS (33\%) as compared to IFI-CNS (10\%) $(P=0.028)$.

The overall response rate (ORR) to treatment was 57\% (with CR 34\% and PR 23\%), without significant differences between
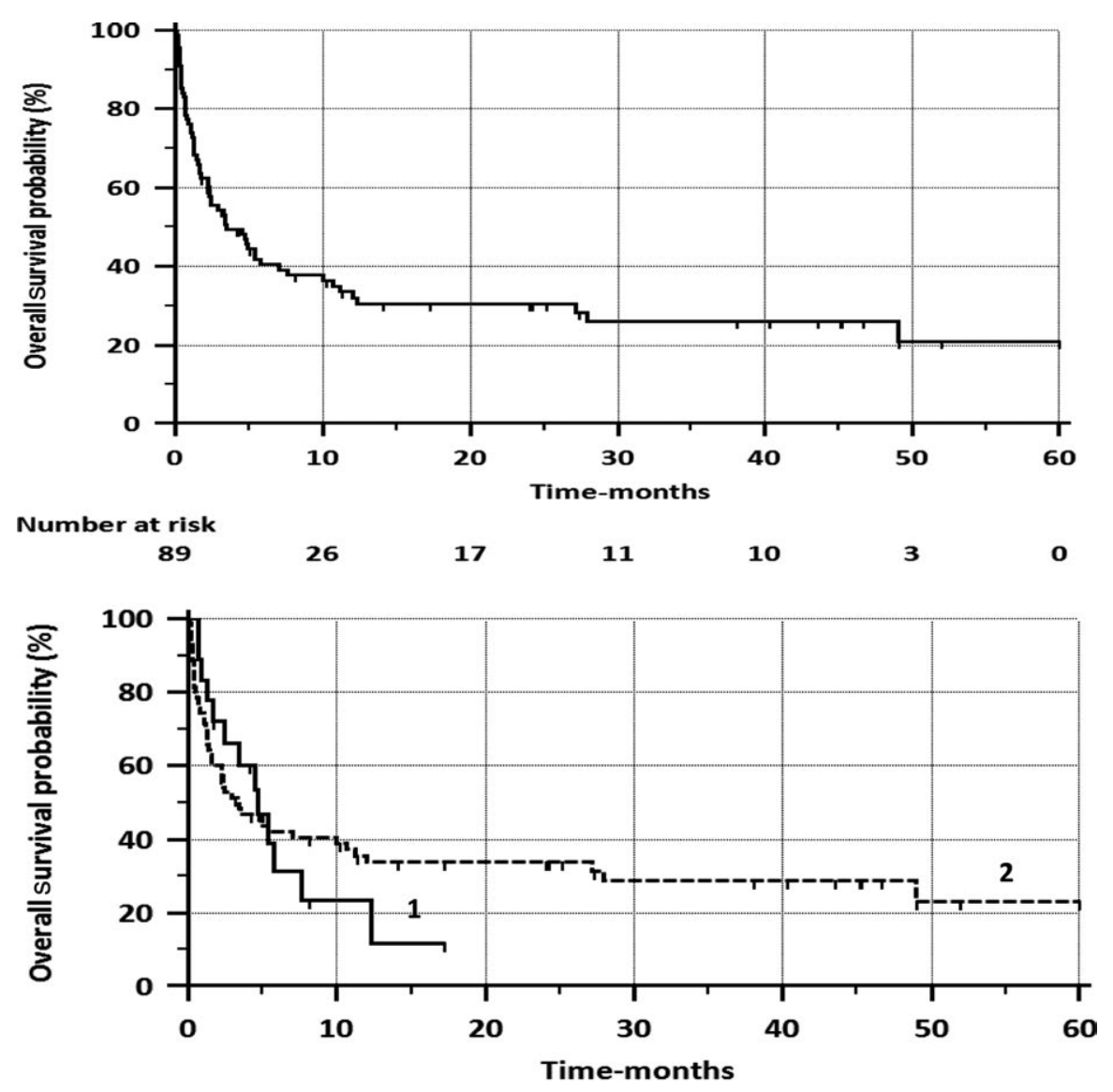

Number at risk

Group: 1 PNS

18

Group: 2 CNS

71

2

24 o

17 o

11 o

10 $\mathbf{0}$

3 o

o
FIGURE 1 (A) Overall Survival from IFI Diagnosis-All Cases. (B) OS from IFI Diagnosis-according to site (CNS or PS) 
the 2 cohorts (Table 3). The ORR in the cases of Aspergillosis and Mucormycosis was $53 \%$ and $52 \%$, respectively.

\section{5 | Outcome}

Median survival of the entire patient population was 3.5 months after the diagnosis of IFI (IFI-CNS 3,3 mths and IFI-PS 4,7 mths) with a 12month OS probability of $32 \%$ (Figure $1 \mathrm{~A}$ ). The 12 -month OS was $23 \%$ in IFI-CNS and 34\% in IFI-PNS (Log-rank, $P>0.05$ ) (Figure 1B). The overall mortality was $69 \%$ (61/89) without a significant difference between the two groups. The mortality rate for IFI as the primary cause (attributable mortality) was 33\% (20/61 cases) while the mortality from haematological disease with IFI as a contributory factor was 44\% (27/61) (Table 3).

Univariate and multivariate analysis of the variables potentially influencing the OS are shown in Table 4. Analyses have been restricted to $66 \mathrm{IFI-CNS}$ that received antimycotic treatment (Table 3). In detail, the following variables were checked and compared: isolated IFI-CNS vs non-isolated forms, proven vs probable IFI, Aspergillosis vs other aetiological agents, PMNs lower or higher than 500 at the diagnosis of IFI, previous Allo-SCT, status of haematologic disease (induction-consolidation vs. refractory disease or relapse), response to antifungal therapy (CR or PR vs not response), use (yes or not) of combination antifungal therapy and surgical intervention (yes or not).
In univariate analysis, the following factors were found to provide a favourable effect on OS: the haematological disease at onset or in consolidation (non-advanced disease) (95\% Cl: 1.007-3.468; $P=0.048$ ) and the response (CR or PR) to antifungal therapy ( $95 \%$ $\mathrm{Cl}$ : 5.081-20.555; $P<0.0001)$. The same variables maintained significance in multivariate analysis $(95 \% \mathrm{Cl}: 1,191-5,507 ; P=0.016 ; 95 \%$ Cl: 6.631-35.652; $P<0.0001$ ) (Table 4).

\section{4 | DISCUSSION}

IFIs with localisation at CNS and PS are a rare and severe infectious complication in the course of haematological diseases. The diagnosis of such infectious complications can be complex because the symptoms and signs are not specific, indirect microbiology techniques are not validated on all biological fluids (i.e, CSF), and biopsy is often difficult in patients with underlying haematological malignancies. ${ }^{1}$ In the cases of CNS or PS-IFI, the antifungal treatment is frequently associated with a poor efficacy and this is due both to the poor permeability of antifungal drugs in anatomical sites (such as CNS) and to the inability, in most cases, to integrate drug therapy with surgery. ${ }^{1,9,12}$

Unfortunately, recent epidemiological studies involving large cohorts of haematological patients with IFI-CNS or IFI-PS are lacking and,

TAB LE 4 Univariate and multivariate analyses of factors that influenced overall survival in 66 cases of IFI-CNS who received treatment

\begin{tabular}{|c|c|c|c|c|}
\hline & SE & $\mathbf{P}$ & Exp & $95 \% \mathrm{Cl}$ \\
\hline $\begin{array}{l}\text { Status of HD (Induction-consolidation vs refractory } \\
\text { or relapse) }\end{array}$ & 0.317 & $\underline{0.048}$ & 1.869 & $1.0072-3.4681$ \\
\hline Response to antifungal therapy (CR/PR vs NR) & 0.3583 & $\leq 0.0001$ & 10.2201 & $5.0814-20.5557$ \\
\hline Proven vs probable IFI & 0.3049 & 0.338 & 0.7468 & $0.4121-1.3534$ \\
\hline Isolated IFI-CNS vs non-isolated forms & 0.3418 & 0.403 & 0.752 & $0.3862-1.4643$ \\
\hline Age at IFI-CNS diagnosis (< or $>65 y$ ) & 0.3922 & 0.1564 & 1.7434 & $0.8114-3.7457$ \\
\hline $\mathrm{PMN}(<\mathrm{or}>500 / \mathrm{mmc})$ & 0.3035 & 0.849 & 1.0591 & $0.5860-1.9143$ \\
\hline Allo-SCT (yes or not) & 0.3156 & 0.195 & 0.6646 & $0.3591-1.2298$ \\
\hline Surgical resection (yes or not) & 0.3618 & 0.26 & 1.503 & $0.7423-3.0434$ \\
\hline Response to antifungal therapy (CR/PR vs NR) & 0.4313 & $\leq \underline{0.0001}$ & 15.3755 & $6.6310-35.6520$ \\
\hline Proven vs probable IFI & 0.4848 & 0.7513 & 0.8575 & $0.3332-2.2072$ \\
\hline Causative agent (Aspergillus vs Others) & 0.5498 & 0.4177 & 1.5613 & $0.5345-4.5609$ \\
\hline Isolated IFI-CNS vs non-isolated forms & 0.3841 & 0.409 & 1.3732 & $0.6493-2.9043$ \\
\hline Age at IFI-CNS diagnosis (< or $>65 \mathrm{y}$ ) & 0.5183 & 0.7727 & 0.8609 & $0.3134-2.3654$ \\
\hline $\mathrm{PMN}(<\mathrm{or}>500 / \mathrm{mmc})$ & 0.3463 & 0.0825 & 1.8246 & $0.9287-3.5848$ \\
\hline Allo-SCT (yes or not) & 0.3777 & 0.485 & 1.3018 & $0.6232-2.7191$ \\
\hline Surgical resection (yes or not) & 0.4996 & 0.3171 & 1.6485 & $0.6222-4.3674$ \\
\hline
\end{tabular}

HD, haematologic disease; Allo-SCT, allogeneic stem cell transplant; PMN, granulocytes. 
therefore, there are no established diagnostic-therapeutic approaches to this serious infectious complication particularly in patients with haematological malignancies although, more recently, attempts have been made to define guidelines based on experts' opinion. ${ }^{12}$

This study shows that, even in the most recent years, the IFI of CNS and PS are a very rare infectious complication in haematological patients. In fact, in the 34 centres participating in this study, less than 1 case per centre per year was observed. With regard to the underlying haematological disease, most of the cases were observed in patients with AML and ALL or in recipients of an allogeneic SCT. ${ }^{1,17,18}$ In most cases, patients had an active disease at the time of the onset of IFI while few cases occurred with a haematological disease in remission or during the consolidation phase. Although $74 \%$ of patients were neutropenic, severe neutropenia (PMN $<500 / \mathrm{mmc}$ ) has been documented in less than $50 \%$ of cases at the time of diagnosis of IFIs. This confirms that, in addition to neutropenia, other factors, such as the active state of the underlying haematological disease and the degree of patient's immunodepression, play an important predisposing role. ${ }^{1}$

We also confirm that IFI-CNS are frequently associated with concomitant involvement of other sites (for instance in the lung). In fact, isolated IFI-CNS forms accounted for only $25 \%$ of the cases in this study. This confirms the aetiopathogenic hypothesis that the IFI-CNS could originate from the haematogenous dissemination of an infection arising from another primary site (such as the lung). ${ }^{1,11,19}$ In this study, a pulmonary IFI has preceded a CNS or PS localisation in $48 \%$ of cases. Therefore, in patients with pulmonary mycosis, the persistence of fever, despite a pulmonary improvement, or the onset of a headache or other neurological signs, such as diplopia, should never be underestimated. With regard to the diagnosis of IFIs, our data show that indirect methods of diagnosis are now commonly used including radiological examinations and biological markers such as GM, which represents a well consolidated diagnostic method. ${ }^{1,9,12,20-24} \beta$-D-Glucan appears not to be widely used, both for blood and CSF. The histological diagnosis remains rarely used, even in the recent period of this study (histological diagnosis was made only in $29 \%$ of cases). In addition, it should be noted that, while diagnostic biopsy of PS was performed in $69 \%$ of cases, brain diagnostic biopsy was performed only in $10 \%$ of IFI-CNS and mainly in patients with a platelet count $>100,000 / \mathrm{mmc}$.

In our series of IFI-CNS, while serum GM was systematically performed, GM on CSF was tested in a limited percentage (42\%) of cases, but in cases where this test was performed, it was positive and, therefore, informative in $67 \%$ of cases. This information underlines that the determination of GM in CSF can be useful in the diagnosis of CNSAspergillosis, and therefore, a diagnostic lumbar puncture should be encouraged in cases with a suspected CNS fungal infection. ${ }^{1,20,25}$ On the other hand, we found that $\beta$-D-Glucan has only been occasionally determined on CSF, although this is a very recent epidemiologic study. This indirect diagnostic test on the CSF should, therefore, be useful, also in accordance with recent literature results. 1,9,12,22,25

From a therapeutic point of view, the study confirms that, even in the most recent years, surgical treatment has been used in a minority of cases, both in the forms of IFI-PS (surgery in 33\% of cases) and especially in the IFI-CNS (surgery only in $10 \%$ of cases). This low percentage of surgery is similar to that of older studies, and it is probably related to various factors such as the presence of disseminated infection, the localisation of the lesion and the high surgical risk related to severe and prolonged thrombocytopenia and immunopression that make these patients at high risk of post-operative infections and bleeding. ${ }^{1,26}$

However, compared to older studies, we have observed an improvement in the response to therapy with an ORR of $57 \%$ (IFI-CNS ORR $=54 \%$, IFI-PS ORR $=67 \%$ ), and this is probably due to the availability of drugs, such as VORI and AMB, able to penetrate well into the CNS and PS. In fact, the most commonly used therapeutic agents in our series were AMB as the first-line treatment (followed by VORI) and VORI as second line (followed by AMB). Additionally, a non-negligible percentage of cases $(38 \%)$ received combination antifungal therapy.

Unfortunately, the median OS of treated cases was short (3.5 months) with a 12-month OS of 32\%. Sixty-nine per cent (61/89) of patients died, but it should be emphasised that many patients died from the underlying haematological disease as the primary cause of death. ${ }^{12,27-29}$ In fact, the attributable mortality was lower (33\%) respect to older studies. Another interesting observation that comes from this study is that we did not find significant differences in terms of attributable mortality when comparing IFI-PS with those of CNS. In univariate and multivariate analyses (performed for the larger cohort of patients with IFI-CNS), the factors that were found to affect OS are the response (CR or PR) to antifungal treatment and the status of haematologic disease (not in an advanced stage).

In conclusion, despite the limitations of a retrospective observational study, this is one of the largest recent epidemiological series of IFI-CNS and PS in haematological patients. This study shows that these infectious complications still have a high mortality rate. However, compared to older epidemiological data, there is a substantial improvement in the response rate to treatment (from less than $20 \%$ to more than $50 \%$ ) and in patient outcome (overall mortality from $100 \%$ to $69 \%$ ). ${ }^{3,30,31}$ There is likely to be further room for improvement, taking into account the current availability of new antifungal drugs with a broad spectrum of action and with penetrability in PS and CNS, such as isavuconazole and the available possible option of combination antifungal therapy. ${ }^{9,32,33}$ Furthermore, the improvement of the diagnostic approach with the execution, in cases of suspected brain mycosis, of a diagnostic lumbar puncture with GM and $\beta$-D-Glucan tests in CSF samples (or new PCR assay) could allow a better and faster diagnostic definition and consequently a timely, targeted and, therefore, more effective, therapy. ${ }^{1,12,20,25,34}$

\section{CONFLICT OF INTEREST}

Dr A. Candoni, Dr A Busca, Dr L Pagano and Dr F. Aversa participated in advisory boards and/or received speaker honoraria from Gilead, Merck, Pfizer, Basilea.

\section{ORCID}

A. Candoni iD https://orcid.org/0000-0003-4436-1310 


\section{REFERENCES}

1. Schwartz S, Kontoyiannis DP, Harrison T, Runke M. Advances in the diagnosis and treatment of fungal infections of the CNS. Lancet Neurol. 2018;17:362-372.

2. Ruhnke M, Kofla G, Otto K, Schwartz S. CNS aspergillosis. CNS Drugs. 2007;21:659.

3. Pagano L, Caira M, Falcucci P, Fianchi L. Fungal CNS infections in patients with hematologic malignancy. Expert Rev Anti Infect Ther. 2005;3:775-785.

4. Scully EP, Baden LR, Katz JT. Fungal brain infections. Curr Opin Neurol. 2008;21:347-352.

5. Gamaletsou MN, Sipsas NV, Roilides E, Walsh TJ. Rhino-orbitalcerebral mucormycosis. Curr Infect Dis Rep. 2012;14:423-434.

6. Jacobs CS, Etherton MR, Lyons JL. Fungal infections of the central nervous system. Curr Infect Dis Rep. 2014;16(12):449.

7. Kontoyiannis DP, Lewis RE. How I treat mucormycosis. Blood. 2011;118:1216-1224.

8. Chakrabarti A, Denning DW, Ferguson BJ, et al. Fungal rhinosinusitis: a categorization and definitional schema addressing current controversies. Laryngoscope. 2009;119(9):1809-1818.

9. Economides MP, Ballester LY, Kumar VA, et al. Invasive mold infections of the central nervous system in patients with hematologic cancer or stem cell transplantation (2000-2016): uncommon, with improved survival but still deadly of ten. J Infect. 2017;75(6):572-580.

10. Messina JA, Maziarz EK, Spec A, Kontoyiannis DP, Perfect JR. Disseminated cryptococcosis with brain involvement in patients with chronic lymphoid malignancies on ibrutinib. Open Forum Infect Dis. 2017;4(1):ofw261.

11. Schwartz S, Reisman A, Troke PF. The efficacy of voriconazole in the treatment of 192 fungal central nervous system infections: a retrospective analysis. Infection. 2011;39:201-210.

12. Schmidt-Hieber M, Silling G, Schalk E, et al. CNS infections in patients with hematological disorders (including allogeneic stem.cell transplantation)-Guidelines of the Infectious Disease Working Party (AGIHO) of the German Society of Hematology and Medical Oncology (DGHO). Ann Oncol. 2016;27(7):1207-1225.

13. Kleinschmidt-DeMasters BK. Central nervous system aspergillosis: a 20-year retrospective series. Hum Pathol. 2002;33:116-124.

14. Farmakiotis D, Kontoyiannis DP. Mucormycoses. Infect Dis Clin North Am. 2016;30(1):143-163.

15. Tortorano AM, Richardson M, Roilides E, et al. ESCMID and ECMM joint guidelines on diagnosis and management of hyalohyphomycosis: Fusarium spp., Scedosporium spp. and others. Clin Microbiol Infect. 2014;20(Suppl. 3):27-46.

16. De Pauw B, Walsh TJ, Donnelly JP, et al. Revised definitions of invasive fungal disease from the European Organization for Research and Treatment of Cancer/Invasive Fungal Infections Cooperative Group and the National Institute of Allergy and Infectious Diseases Mycoses Study Group (EORTC/MSG) Consensus Group. Clin Infect Dis. 2008;15(46):1813-1821.

17. Baddley JW, Salzman D, Pappas PG. Fungal brain abscess in transplant recipients: epidemiologic, microbiologic, and clinical features. Clin Transplant. 2002;16:419-424.

18. Balaguer Rosello A, Bataller L, Lorenzo I, et al. Infections of the central nervous system after unrelated donor umbilical cord blood transplantation or human leukocyte antigen matched sibling transplantation. Biol Blood Marrow Transplant. 2017;23(1):134-139.

19. Higo T, Kobayashi T, Yamazaki S, et al. Cerebral embolism through hematogenous dissemination of pulmonary mucormycosis complicating relapsed leukemia. Int J Clin Exp Pathol. 2015;8(10):13639-13642.

20. Lyons JL, Zhang SX. Current laboratory approaches to diagnosis of CNS fungal infections. Future Microbiol. 2016;11(2):175-177.
21. Marchetti O, Lamoth F, Mikulska M, Viscoli C, Verweij P, Bretagne S; European Conference on Infections in Leukemia (ECIL) Laboratory Working Groups. ECIL recommendations for the use of biological markers for the diagnosis of invasive fungal diseases in leukemic patients and hematopoietic SCT recipients. Bone Marrow Transplant. 2012;47:846-54.

22. Mikulska M, Furfaro E, Del Bono V, et al. (1-3)- $\beta$-d-glucan in cerebrospinal fluid is useful for the diagnosis of central nervous system fungal infections. Clin Infect Dis. 2013;56:1511-1512.

23. Viscoli C, Machetti M, Gazzola P, et al. Aspergillus galactomannan antigen in the cerebrospinal fluid of bone marrow transplant recipients with probable cerebral aspergillosis. J Clin Microbiol. 2002;40:1496-1499.

24. Swinburne NC, Bansal AG, Aggarwal A, Doshi AH. Neuroimaging in central nervous system infections. Curr Neurol Neurosci Rep. 2017;17(6):49.

25. McCarthy MW, Walsh TJ. Molecular diagnosis of invasive mycoses of the central nervous system. Expert Rev Mol Diagn. 2017;17(2):129-139.

26. Mattiuzzi G, Giles FJ. Management of intracranial fungal infections in patients with haematological malignancies. $\mathrm{Br} J$ Haematol. 2005;131:287-300.

27. Wiederhold NP, Pennick GJ, Dorsey SA, et al. A reference laboratory experience of clinically achievable voriconazole, posaconazole, and itraconazole concentrations within the bloodstream and cerebral spinal fluid. Antimicrob Agents Chemother. 2014;58(1):424-431.

28. Groll AH, Giri N, Petraitis V, et al. Comparative efficacy and distribution of lipid formulations of amphotericin B in experimental Candida albicans infection of the central nervous system. J Infect Dis. 2000;182:274-282.

29. Tissot F, Agrawal S, Pagano L, et al. ECIL-6 guidelines for the treatment of invasive candidiasis, aspergillosis and mucormycosis in leukemia and hematopoietic stem cell transplant patients. Haematologica. 2017;102(3):433-444.

30. Dragonetti G, Criscuolo M, Fianchi L, Pagano L. Invasive aspergillosis in acute myeloid leukemia: are we making progress in reducing mortality? Med Mycol. 2017;55(1):82-86.

31. Pagano L, Ricci P, Montillo M, et al. Localization of aspergillosis to the central nervous system among patients with acute leukemia: report of 14 cases. Gruppo Italiano Malattie Ematologiche dell'Adulto Infection Program. Clin Infect Dis. 1996;23(3):628-630.

32. Pagano L, Cornely OA, Busca A, et al. Combined antifungal approach for the treatment of invasive mucormycosis in patients with hematologic diseases: a report from the SEIFEM and FUNGISCOPE registries. Haematologica. 2013;98(10):e127-e130.

33. Candoni A, Aversa F, Busca A, et al. Combination antifungal therapy for invasive mould diseases in haematologic patients. An update on clinical data. J Chemother. 2015;27(1):1-12.

34. Reinwald $M$, Buchheidt $D$, Hummel $M$, et al. Diagnostic performance of an Aspergillus-specific nested PCR assay in cerebrospinal fluid samples of immunocompromised patients for detection of central nervous system aspergillosis. PLoS ONE. 2013;8(2):e56706.

How to cite this article: Candoni A, Klimko N, Busca A, et al. ; on behalf of SEIFEM Group (Epidemiological Surveillance of Infections in Haematological Diseases). Fungal infections of the central nervous system and paranasal sinuses in onco-haematologic patients. Epidemiological study reporting the diagnostic-therapeutic approach and outcome in 89 cases. Mycoses. 2019;00:1-9. https://doi.org/10.1111/ myc.12884 